\title{
Towards Quality Control of Food Using Terahertz
}

\author{
B. S. -Y. Ung and B. M. Fischer and B. W. -H. Ng and D. Abbott \\ Centre for Biomedical Engineering and School of Electrical \& Electronic Engineering, \\ The University of Adelaide Adelaide, SA 5005, Australia
}

\begin{abstract}
Terahertz radiation or T-rays, show promise in quality control of food products. As T-rays are inherently sensitive to water, they are very suitable for moisture detection. This proves to be a valuable asset in detecting the moisture content of dried food, a critical area for some products. As T-rays are transparent to plastics, food additives can also be probed through the packaging, providing checks against a manufacturer's claims, such as the presence of certain substances in foods.
\end{abstract}

Keywords: moisture content, terahertz spectroscopy, T-rays

\section{INTRODUCTION}

Terahertz radiation or T-rays show much promise in improvements to personal health and security. As many non-polar and non-metallic substances are transparent to T-rays, ${ }^{1-3}$ and T-rays are sensitive to molecular bonds, ${ }^{2}$ this opens the possibility of substance detection. T-rays lie between the microwave and infrared bands of the electromagnetic spectrum and are non-ionising, giving T-rays the ability to scan or probe substances without damaging them. These abilities suggest that T-rays are able to be used within the food industry providing forms of quality assurance and control. T-rays can be used in varying applications of time domain spectroscopy (THz-TDS) or imagining to achieve the desired results. ${ }^{1-6}$

\section{MOTIVATION}

Food production in developing countries, especially in Asia are under increasing scrutiny to produce safe edible foods. The demand for food is ever increasing, and food quality assurance and control is required for the manufacturers to establish brand recognition and to produce safe foods for public consumption. This requires checks and testing of foods to ensure that they are in fact safe for public consumption. Current tests are unable to detect contaminants within packaging and also foreign particles (millimetre scale or less ${ }^{7}$ ) hidden within food. Moisture content in food is also of question, as the weight of food is increased if there is a higher moisture content. This is of particular concern with large quantities of dried foods, which could lead to significant changes to a company's profitability and reputation. Thus new methods are needed in this case to increase safe food quality and production. T-rays prove to be a usable and valid technique for this.

\section{CURRENT WORK}

The current work in T-ray food quality control shows much promise, especially the published material on chocolate, ${ }^{7}$ wheat $^{8}$ and aqueous alcohol and sugar solutions. ${ }^{9}$ Investigations into ripeness of tomatoes have also been investigated by Ogawa et al., however, the implications of the experimentation shows less promise.

Quality assurance of chocolate products conducted by Joerdens et al. ${ }^{7}$ presents how T-rays are able to detect foreign particles in blocks of chocolate. Stones or glass splinters of the scale of mm were readably discernible in line scans that were taken. This can be seen in Figure 1, where the line scan of contaminated chocolate shows distinct differences to chocolate that is free of contaminants. As the refractive indexes of the stone and glass particles are higher than that of chocolate, the line scans show double-pulses, while plain chocolate and chocolate containing nuts show no identifiable change in the shape of the pulse, due to nuts having a similar refractive index to chocolate.

Further author information: (Send correspondence to B. Ung)

B. Ung: E-mail: bung@eleceng.adelaide.edu.au

BioMEMS and Nanotechnology III, edited by Dan V. Nicolau, Derek Abbott, Kourosh Kalantar-Zadeh, Tiziana Di Matteo, Sergey M. Bezrukov, Proc. of SPIE Vol. 6799, 67991E, (2007) · 0277-786X/07/\$18 · doi: 10.1117/12.759825 


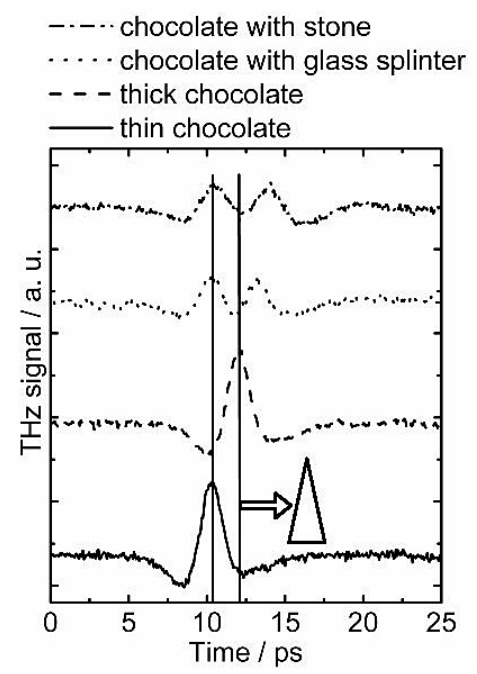

Figure 1. Line scans of chocolate of thin and thick thicknesses, and chocolate contaminanted with stones and glass shards. After Joerdens et al. ${ }^{7}$

As T-rays are highly water absorbant, they are very useful in sensitive detection of water content of substances. Chua et al. ${ }^{8}$ used this property to perform THz-TDS on wheat to determine the level of moisture content. The frequency range of 0.1 to $4.0 \mathrm{THz}$ was used to examine whole and crushed wheat with moisture contents of $8 \%, 12 \%$ and $18 \%$. Analysis of the whole wheat grain showed no conclusive results, as the whole wheat grain exhibited scattering and was highly dependent on the orientation relative to the incident T-ray beam. The results given show a frequency range from 0.1 to $1 \mathrm{THz}$, whereby the distinct water lines at $0.32,0.50,0.75$, and $0.92 \mathrm{THz}$ within this range are not apparent and do not distinctly show the difference between the levels of moisture content in the wheat grain. When the wheat was crushed, the spectra was free from the scattering of the whole wheat grain, and showed tenable results, with distinct water lines shown in the $18 \%$ moisture content. This can be seen in Figure 2, where the frequency range shown from 0.1 to $2.50 \mathrm{THz}$ shows the varying levels of absorption of the moisture content of the crushed wheat grain.

Unlike measurements of chocolate and wheat, the measurement of aqueous ethanol and sucrose solutions was performed in reflection mode rather than transmission mode. ${ }^{9}$ This presented challenges for the analysis, as complex equations were required to obtain the absorption coefficients of the solutions. Measurements were made on 21 samples of alcohol water mixtures, with alcohol contents ranging from $0 \%$ to $100 \%$, in $5 \%$ percent increments and also aqueous sucrose solutions, ranging from $0 \%$ to $75 \%$ concentration by weight. Jepsen et al. were able to determine the concentration of alcohol in an unknown alcohol water mixture, by comparing the dielectric function produced from experimentation of known quantities of alcohol. This then lead to the determination of alcohol content in commercial beverages, by use of the same method. Additionally, Jepson et al. found that the accuracy of results were best while the concentrations of alcohol were low, as the signal to noise ratio was higher than that with higher alcohol concerntrations.

\section{POSSIBLE FUTURE WORK}

Currently, simple time domain analysis and THz-TDS in both transmission and reflection may be used for food quality control. Imaging however is relatively untapped, offering possibilities to image at differing terahertz frequencies provides the possibility to quickly analyse and determine if foreign objects are located within a food's packaging or within the food itself. Clear absorption peaks of substances within the terahertz frequency range would indeed make this easier to develop algorithms to self automate quality control systems. These techniques could provide additional methods to determine moisture content in other food types, and spectroscopy could 


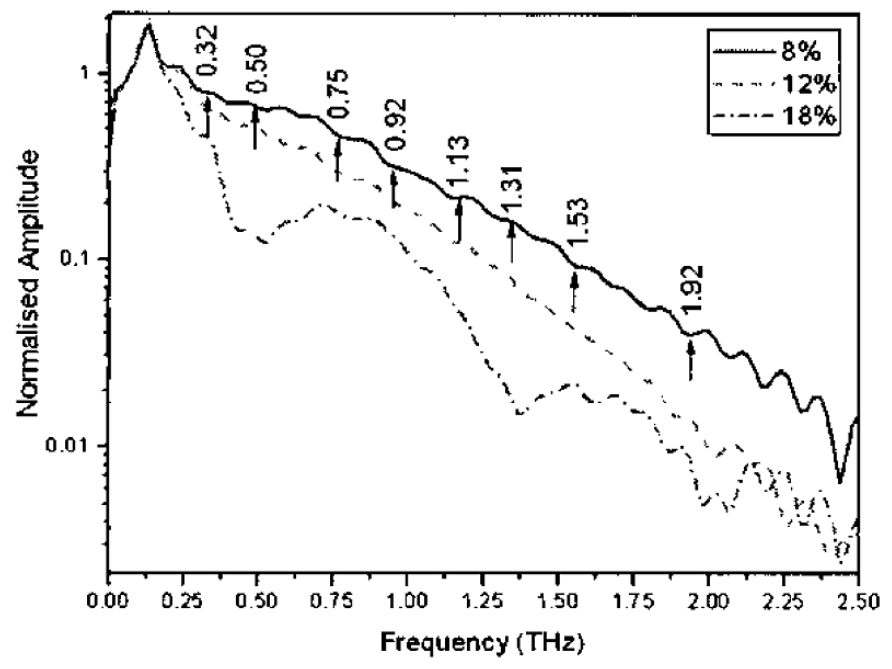

Figure 2. Transmission spectra of the crushed wheat, showing water absorption lines for crushed wheat grain with water contents of $8 \%, 12 \%$ and $18 \%$. After Chua et al. ${ }^{8}$

determine if there are any harmful chemicals present in foods without the need to remove food from its packaging, which could lead to faster test and if necessary recall times of foods.

The current methods of use of T-rays are not without constraints. As mentioned previously, scattering of the T-rays in both transmission and reflection of the incident T-ray beam is a major problem. ${ }^{10}$ This is especially apparent in the whole wheat grain, ${ }^{8}$ where the normalised transmission spectra shows no tangible results and is highly dependent on the orientation of the wheat grain to the incident T-ray. Penetration of T-rays in transmission is also a problem, particularly with water. ${ }^{9}$ Currently, T-rays are used in reflection mode in order to perform THz-TDS.

Clever algorithms to remove scattering are required to account for problems that are encountered during the measurement process, and to obtain useable data. ${ }^{1}$ Penetration depth of aqueous solutions could be solved by increasing the power of the transmitted T-ray beam, which would remove the need to operate in reflection mode, removing polarization problems and the need for complex analysis. Finally, the scan speed using T-rays is currently slow, this is particularly the case when performing T-ray imaging. ${ }^{5,6}$ If the speed of the scans can be improved for real-time analysis, T-rays could very well be used by many manufacturers in automated food quality control.

\section{REFERENCES}

1. D. M. Mittleman, M. Gupta, R. Neelamani, R. G. Baraniuk, J. V. Rudd, and M. Kock, "Recent advances in terahertz imaging," Applied Physics B - Lasers and Optics 68, pp. 1085-1094, 1999.

2. B. Fischer, M. Hoffmann, H. Helm, G. Modjesch, and P. U. Jepsen, "Chemical recognition in time-domain spectroscopy and imaging," Semiconductor Science and Technology 20, pp. 246-253, 2005.

3. W. Withayachumnankul, G. Png, X. Yin, S. Atakaramians, I. Jones, H. Lin, B. Ung, J. Balakrishnan, B. Ng, B. Ferguson, S. Mickan, B. Fischer, and D. Abbott, "T-ray sensing and imaging," Proceedings of the IEEE 9, pp. 1528-1558, 2007.

4. D. Abbott and X.-C. Zhang, "T-ray imaging, sensing, and retection," Proceedings of the IEEE 9, pp. 15091513, 2007.

5. B. Fergusson and D. Abbott, "Wavelet de-noising of optical terahertz pulse imaging data," Fluctuation and Noise Letters 1, pp. 65-69, 2001. 
6. S. Mickan, D. Abbott, J. Munch, X. C. Zhang, and T. van Doorn, "Analysis of system trade-offs for terahertz imaging," Micro Electronics Journal 31, pp. 503-514, 2000.

7. C. Joerdens, F. Rutz, T. Hasek, and M. Koch, "Towards real-time terahertz quality assurance of chocolate products," Infrared Millimeter Waves and 14th International Conference on Teraherz Electronics, 2006. IRMMW-THz 2006. , pp. 332-332, 2006.

8. C. Joerdens, F. Rutz, T. Hasek, and M. Koch, "Terahertz time-domain spectroscopy of wheat grain," Infrared and Millimeter Waves, 2004 and 12th International Conference on Terahertz Electronics, 2004. , pp. 399-400, 2004.

9. P. U. Jepson, U. Moller, and H. Merbold, "Investigation of aqueous alcohol and sugar solutions with reflection terahertz time-domain spectroscopy," Optics Express 15, pp. 14717-14737, 2007.

10. P. Swift, D. Chang, J. Fletcher, A. Gallant, J. Levitt, R. Abram, D. Beggs, M. Kaliteevski, and J. Chamberlain, "Terahertz scattering: Comparison of a novel theoretical approach with experiment," Proceedings of the SPIE, Terahertz and Gigahertz Electronics and Photonics V, pp. 186-194, 2006. 